\title{
Studii sui punti singolari essenziali delle funzioni analitiche di due o più variabili complesse.
}

\author{
(Di Euaenio Euad Lev, a Genova.)
}

\section{$\$ I$.}

1. Sia $f(x y)$ una funzione monodroma (*) analitica di due variabili complesse $x=x_{1}+i x_{2}, y=y_{1}+i y_{2}$. Si dice che $f(x y)$ c̀ regolare nel punto $(\xi, n)$ se nell'intorno di questo punto è sviluppabile in serie di potenze di $x-\xi, y-n$; si dice che in $(\xi, n)$ ha un pninto singolare non essenziale, se è possibile trovare una serie di potenze $p_{0}\left(x-\xi, y-x_{1}\right)$ tale che il prodotto $p_{0}(x-\xi, y-n) f(x y)$ sia regolare nel punto $\left(\xi, x_{1}\right)$; e ciò̀ quando la $f(x y)$ si può porre nella forma $f(x y)=\frac{p_{1}\left(x-\xi, y-n_{1}\right)}{p_{0}\left(x-\xi, y-n_{1}\right)}$ dove $p_{1}(x-\xi, y-n)$ indica essa pure una serie di potenze di $x-\zeta, y-n$. Ogni altro punto sarà per la funzione un punto singolare essenzinle (**).

Nel presente lavoro mi propongo di mostrare che le varictà formate dai punti singolari essenziali soddisfanno a condizioni assai restrittive; o, il che è lo stesso, che i campi in cui una funzione può essere meromorfa $\left(^{* * *}\right)$ sono di natura particolare. Così verrà mostrato che è erronea l'affermazione del Wennstrass che, analogamente a quanto arviene per le funzioni di una sola

(*) Sovente per la validità di quanto segue si potrà anche considerare un ramo monodromo di una funzione polidroma.

${ }^{(* *)}$ Così sono punti singolari essenziali anche quelli di un campo (lacunare) cui non si possa giungere per prolungamento analitico.

(***) $\mathrm{E}$ cioè in cui la funzione può non avere punti regolari e punti singolari non essenziali : secondo WeIERSTRASS si direbbe: in cui una funzione può avere comportamento ali una funzione razionale. 
variabile, assegnato ad arbitrio un campo continuo a 4 dimensioni nello spazio in cui sono rappresentate le variabili complesse $x y$, esiste sempre una funzione meromorfa in esso, che ha singolarità essenziali in ogni punto del contorno di questo $\left(^{*}\right)$.

La natura degli insiemi di punti singolari di una funzione analitica ̀̀ già stata studiata dall'Hantogs (**) nei suoi bei lavori partendo sia dalla formula di $\mathrm{C}_{A U G H Y}$ sia dagli sviluppi in serie di potenze: però l'HarTogs non riesce a scindere nelle sue considerazioni i punti singolari essenziali dai non essenziali, cosicchè i suoi risultati nulla ci dicono sui punti singolari essenziali limiti di punti singolari non essenziali; e limitano la natura dei campi in cui la funzione può essere regolare, e non di quelli in cui può essere meromorfa.

Le deduzioni di questo lavoro hanno comune fondamento nel semplicissimo teorema del $\S I(n .4$ e 5) secondo il concetto esposto nel n. 6 . Nel \$II mostro come da questo solo principio e nel campo più ampio ora ricordato si possano facilmente dedurre tutti i principali risultati che l'Hartogs ottenne per varie vie. Nel \& III determino alcune proprietà differenziali delle ipersuperficie che possono costituire la frontiera di un campo in cui una funzione analitica di due variabili complesse è meromorfa.

Tutti i risultati di questo lavoro si estendono senza difficoltà essenziali alle funzioni di più variabili.

2. Un'equazione $p(x y)=0$, dove $\varphi$ è una funzione analitica di $x$ ed $y$, determina nello spazio a 4 dimensioni, in eui si rappresentano i punti $(x, y)$, una varietà a due dimensioni di natura particolare: indicherò queste varietà

(*) Wennatrass, Untersuchungen über die $2 r$ fach periodischen Functionen von $r$ Verönderlichen. Grelles Jourual, 89, Weeke II, pag. 123-133: Wird aus dem Gehicte von $r$ complexen Veränderlichen $u_{1} u_{2} \ldots u_{r}$ auf irgend eine Weise ein $2 r$-fach ausgedehntrs Continuum ausgeschieden, so lassen sich stets eindeutige Functionen von $u_{1}, \ldots u_{r}$ bestimmen, welche sich an allen Stellen im Innern dieses Continuums, aber an keiner Stelle seiner Begrenzung wie rationale Functionen verhalten.

(**) Fritz HARTOGs : $-1{ }^{\circ}$ Zur Theorie der analytischen Functionen mehrerer unabhängiger Veränderlichen, otc. Math. Annalen, Bd. 62, pag. 1-88. - 2. Einige Folgerungen aus der Cauchy'schen Integralfornel bei Funktionen mehrere. Verändenlichen. Milnchener Sitzungsberichte, Bd. 36 (1906) - 3. ${ }^{\circ}$ Veber die aus den singulären Stellen einer analytischen Funktion mehreror Veränderlichen bestehenden Gebiete. Acta Mathematica, Vol. 32 (1909), pag. 57-79. - Vedi anche il rapporto riassinnlivo dell'HaR'rogs nei Jahresber. der Deutscher Math. Vereinigung, 16 (1907), pag. 223 e ss. Ueber nenere Untersuchungen auf dem Gebiete der analytischen Funtionen melererer Verändertichen. 
col nome di varietà o superficie caratteristiche $\left(^{*}\right)$ : in particolare un'equazione lineare tra $x$ ed $y$ rappresenta un piano caralteristico.

Un punto di una superficie caratteristica $\phi(x y)=0$ può essere: regolwre o critico se la superficie può nell'intorno di esso essere rappresentata col dare $x($ od $y)$ in serie di potenze intere o fratte di $y(o d x)$; singolare se è un punto singolare di $\stackrel{\imath}{(x y)}$ limite di punti in cui è $\varphi(x y)=0$.

Con queste definizioni risulta che noi potremo parlare di multiplicità di intersezione di un piano caratteristico con una superficie caratteristica in un punto regolare o critico per questa: risulta pure che, data una superficie caratteristica che in $\left(\xi x_{i}\right)$ abbia un punto regolare o critico, esiste un intorno $\sigma$ di $(\xi n)$ sufficientemente piccolo ed un numero $m$ tale che ogni piano caratteristico $x=$ cost. ha in $\sigma$ al più $m$ punti - contato ognuno colla sua molteplicità, - comuni con essa, fatta eccezione per il piano $x=\xi$ che può appartenere alla superficie medesima.

Applichiamo queste considerazioni ai punti singolari non essenziali di una funzione $f(x y)$. Se $\left(\xi, x_{1}\right)$ è un tale punto, e se precisamente nell'intorno di esso è $f(x y)=\frac{p_{1}(x-\xi, y-n)}{p_{0}(x-\xi, y-n)}$, i soli punti singolari di $f(x y)$ che esistono nell'intorno di $\left(\xi_{n}\right)$ sono punti singolari non essenziali i quali appartengono alla varietà caratteristica $p_{0}(x-\xi, y-n)=0:(\xi, n)$ è su essa un punto regolare o critico. Ne segue:

Osservazione $I$. Se $\left(\zeta r_{1}\right)$ è un punto regolare o singolare non essenziale di $f(x y)$ si può trovare un intorno s di esso ad un numero $m$, tale che ogui piano $x=$ cost. abbia in $"$ al più $m$ punti singolari di $f(x y)$ tutti non essenziali, fatta eventualmente eccezione per il piano $x=\zeta$ che può far parte della varietà caratteristica di punti sinģolari non essenziali cui appartiene $\left(\vdots x_{1}\right)$. Infatti se $(\xi \pi)$ è regolare non esistono punti singolari nel suo intorno: si può fare $m=0$. Se $(\xi n)$ è singolare non essenziale è questa un'immediata conseguenza del fatto che i punti singolari di $f(x, y)$ nell'intorno di $\left(\xi r_{0}\right)$ co-

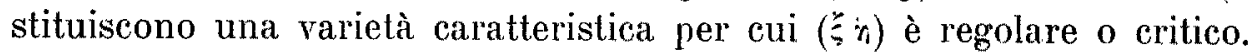

L'osservazione precedente vale pure quando si conti ogni punto ( $\left.\zeta^{\prime} \cdot n^{\prime}\right)$ singolare non essenziale per $f(x y)$ come multiplo secondo il numero che

(*) Seguendo la terminologia proposta dal Levi-Civıta: Sulle funzioni analitiche di due o piu variabili complesse. Rend. della R. Accademia dei Lincei, (1905), vol. XIV, sem. 2, pag. 499-9. Tali varietà sono invero le varietà caratteristiche rispetto al problema di CAUchY per il noto sistema di equazioni cui devono soddisfare la parte reale ed il coefficiente dell'immaginario di una funzione di due varialili. 
rappresenta l'ordine del polo che $f\left({ }^{\prime} y\right)$ ha in $y=n_{i}^{\prime}\left({ }^{*}\right)$. Invero tale ordine è sempre inferiore all'ordine infinitesimale di $p_{0}(x-\xi, y-n)$ in $x=\xi$, $y=n^{\prime}$.

Osservazione II. Se un piano $x=\xi$ non è tutto di punti singolari non essenziali per $f(x y)$, ma su esso esistono infiniti punti singolari non essenziali di $f(x y)$, ogni punto $\left(\begin{array}{r}* \\ \hdashline\end{array}\right)$ limite di questi punti singolari non essenziali è un punto singolare essenziale di $f(x y)$. Infatti tale punto per l'osservazione precedente non può essere nè regolare, nè singolare non essenziale.

3. Aggiungiamo un lemma sullo sviluppo di Launentr.

Lemma. Sia $f(x y)$ una funzione regolare nei punti in cui

$$
|\boldsymbol{x}| \leq \delta, \quad k_{1} \geq|y| \geq k_{2} .
$$

Essa si svilupperà in serie di LAURENT rapporto ad $y$ con coefficienti funzioni analitiche di $x$ per $|x| \leq \delta$ :

$$
f(x y)=\sum_{-\infty}^{\infty} g_{n}(x) y^{n}
$$

Condizione necessaria e sufficiente affinchè questa funzione sia meromorfa nel campo $|x| \leq \delta,|y| \leq k_{1}$ è che esista un numero finito $l+1$ di funzioni - analitiche -- di $x: A_{0}(x), A_{1}(x), \ldots, A_{l}(x)$ tali che sia identicamente per $n<0$ :

$$
A_{0}(x) g_{n-l}(x)+A_{1}(x) g_{n-l+1}(x)+\cdots+A_{l}(x) g_{n}(x)=0
$$

Per maggior chiarezza premettiamo l'osservazione che, se delle funzioni $A_{0}, A_{1}, \ldots, A_{t}$ esistono le quali soddisfano alle (2), esse potranno certamente ritenersi funzioni regolari analitiche di $x$ per $|x| \leq \delta$. Infatti osserviamo che le $g_{n}(x)$ sono funzioni analitiche regolari per $|x| \leq \delta$; e quindi, se le (2) sono equazioni compatibili nelle $A_{i}(x)$, esse si possono risolvere con funzioni analitiche di $x$ regolari per $|x| \leq \delta$.

Ciò posto, la condizione è sufficiente. Infatti la funzione

$$
\psi(x y)=f(x y) \cdot\left[A_{0}(x) y^{I}+A_{1}(x) y^{l-1}+\cdots+A_{i}(x)\right]
$$

sarà come la $f(x y)$ una funzione regolare per $x \leq \delta, k_{1} \supseteq y \geqslant k_{2}$. Ma

(*) È noto che $\mathrm{i}$ soli punti in cui la funzione $f\left(\xi^{\prime} y\right)$ della variabile $y$ può avere un polo sono i punti $(\xi y)$ in cui $f(x y)$ ha una singolarita non essenziale: non riceversa. 
per (1) e (2) essa si svilupperà in questo campo nella forma

$$
\psi(x y)=\sum_{0}^{\infty}\left[A_{0}(x) g_{n-i}(x)+A_{1}(x) g_{n-1+1}(x)+\cdots+A_{l}(x) g_{n}(x)\right] y^{n}
$$

e cioè per sole potenze positive di $y$ : onde segue che essa ̀̀ regolare analitica per $|x| \leq \delta,|y| \leq k_{1}$. Quindi si arră che

$$
f(x y)=\frac{\psi(x y)}{A_{0}(x) y^{\prime}+A_{1}(x) y^{\prime-1}+\cdots+A_{\imath}(x)},
$$

come quoziente di due funzioni analitiche regolari nel campo $|x| \leq \delta,|y| \leq k_{1}$, sarà meromorfa nel campo medesimo.

La condizione è necessaria. Infatti poichè $f(x y)$ è regolare per $|x| \leq \delta$, $k_{1} \supseteq|y| \geqslant k_{2}$, nessun piano $x=x_{0}$ con $\left|x_{0}\right| \leq \delta$ è una varietà di punti singolari non essenziali di $f(x y)$ : onde per l'osservazione II del $n$. 2 su esso esiste nel cerchio $|y| \leq k_{2}$ un numero finito di punti singolari non essenziali. Sia $m_{x_{0}}$ il numero di tali punti sul piano $x=x_{0}$, ognuno contato colla multiplicità che ha il polo di $f\left(x_{0} y\right)$ in esso: $m_{x_{0}}$ avrà per $\left|x_{0}\right| \leq \delta$ un massimo $l$. Infatti ove questo non esistesse, ri sarebbe un punto $(\xi n)$ con $|\xi| \leqslant \delta$ e $|n| \leq k_{2}$ tale che si troverebbero piani $x=x_{0}$ che dentro ad un intorno arbitrariamente piccolo di esso, avrebbero un numero di punti singolari non essenziali superiore ad ogni numero fissato grande a piacere: e ciò contraddice per l'osservazione $I$ del $n .2$ all'ipotesi ehe $\xi n$ sia un punto regolare non essenziale.

Concludendo su ogui piano $x=x_{0}\left(\operatorname{con}\left|x_{0}\right| \leq \delta\right) f\left(x_{0} y\right)$ ha al più $l$ poli, distinti o coincidenti, entro il cerchio $|y| \leqslant k_{2}$ e quindi entro il cerchio $|y| \leq k_{1}$; onde potremo costrurre un'equazione

$$
A_{0}(x) y^{\prime}+A_{1}(x) y^{l-1}+\cdots+A_{l}(x)=0
$$

tale che per ogni valore di $x$ i poli di $f(x y)$ siano tra le radici di essa, ciascuno contato colla sua molteplicità. La funzione

$$
f(x y)\left[A_{0}(x) y^{2}+A_{1}(x) y^{2-1}+\cdots+A_{r}(x)\right]
$$

sàrà quindi su ogni piano $x=$ cost. una funzione della variabile $y$ regolare entro il cerchio $|y| \leq k_{1}$ onde il suo sviluppo per potenze di $y$ deve mancare di potenze negative. E ciò richiede che le $A_{i}(x)$ così costruite soddisfacciano identicamente alle equazioni (2). 
Corollario. Il numero $l$ che compare nel precedente enunciato è un limite superiore per il numero dei punti singolari non essenziali di $f(x y)$ appartenenti ad un piano $x=x_{0}$ con $\left|x_{0}\right|<\delta$.

4. Premesso questo lemma possiamo dimostrare il seguente

Teorema. Sia $f(x y)$ una funzione analitica di $x$ ed $y$ che nel punto (00) abbia una singolarità essenziale, mentre nei punti di un certo intorno $|y| \leq k$ dell'origine appartenenti al piano $x=0$ sia meromorfa. Dato un numero $\&$ piccolo a piacere, è sempre possibile trovare un numero $\delta$ tale che in ogni piano $x=x_{0}$ con $\left|x_{0}\right| \leq \delta$ esista sempre almeno un punto singolare essenziale per $f(x y)$ per cui $\grave{e}|y| \leq \varepsilon\left(^{*}\right)$.

Intanto possiamo supporre che nei punti del piano $x=0$ la funzione sia generalmente regolare: poichè altrinenti i punti generici del piano $x=0$ sarebbero tutti punti singolari non essenziali, e quindi esisterebbe un numero $n$ tale che $x^{n} f(x y)$ avrebbe ancora come $f(x y)$ il punto $(00)$ come punto singolare essenziale, e sarebbe meromorfa, ma generalmente regolare per $x=0, y=1=0$.

Ciò posto le nostre ipotesi dicono che $f(x y)$ può avere dei punti singolari non essenziali nei punti $(0 y)$ con $y=1=0$, ma l'unico punto limite che essi possono avere è, per l'osservazione II del n. 2, il punto $(00)$. Onde fissato $\varepsilon$, potremo trovare nel piano $(0 y)$ una corona circolare di centro l'origine limitata dai cerchi di raggio $k_{1}$ e $k_{2}\left(k_{1}>k_{2}\right)$, interna ad ambedue i cerchi $|y| \leq,|y| \leq \varepsilon$, tale che in qualunque punto di essa e del suo contorno $f(x y)$ sia regolare: onde per il teorema di Laurent nel campo medesimo si potrà sviluppare in serie di potenze positive e negative di $y$ :

$$
f(x, y)=\sum_{-\infty}^{\infty} g_{n}(x) y^{n}
$$

dove le $g_{n}(x)$ sono funzioni analitiche di $x$ per $|x| \leq \delta$. E poichè il punto $(00)$ è un punto singolare essenziale, per il lemma precedente le $g_{n}(x)$ non soddisfanno alcun sistema del tipo (2) per $|x| \leq 0$.

Ne viene che per $\left|x_{0}\right| \leq \delta$ la $f(x y)$ ha sul piano $x=x_{0}$ entro il cerchio $|y| \leq k_{2}-$ e. quindi a fortiori entro il cerchio $|y| \leq \varepsilon-$ almeno un punto singolare essenziale. Invero, ove ciò non fosse, la $f(x y)$ sarebbe meromorfa

(*) Pel caso che $f(x y)$ sia regolare nei punti $x=0,0<|y| \leq k$, questo teorema è stato dimostrato dall'Hartogs, 1 . e. $2 .^{\circ}$; e poi con una dimostrazione del tipo di quella usata nel testo in 1 . c. $3 .^{\circ}$. 
in tutti i punti del piano $x=x_{0},|y| \leq k$, e vi avrebbe un numero finito di punti singolari non essenziali, poichè altrimenti per l'osservazione II del n. 2 esso sarebbe tutto di punti singolari non essenziali il che è contrario all'ipotesi che per $x=x_{0}, k_{1} \geqslant|y| \geqslant k_{2}, f(x y)$ sia regolare. Quindi esisterebbe un campo $\left|x_{0}-x\right| \leq \delta^{\prime},|y| \leq k_{1}$ in cui la funzione $f(x y)$ arrebbe solo un numero finito di punti singolari non essenziali su ogni piano $x=$ cost.: e quindi si svilupperebbe in una serie di Laurest :

$$
f(x y)=\sum_{-\infty}^{\infty} y_{i}^{\prime}(x) y^{\prime \prime}
$$

le $g^{\prime}{ }_{n}(x)$ essendo funzioni regolari analitiche di $x$ per $\left|x-x_{0}\right| \leq \delta^{\prime}$ : e per il lemma del $n$. precedente esisterebbe un numero $l$ tale che le $g_{n}^{\prime}(x)$ soddisfanno (per $n<0$ ) ad un sistema del tipo (2). Ma poichè i campi $\left|x-x_{0}\right| \leq \delta^{\prime}$, $k_{1} \supseteq|y| \geqslant k_{2}$, e $|x| \leqslant \delta, k_{1} \geqslant|y| \geqslant k_{2}$ hamo un campo a quattro dimensioni comune, i due sviluppi (4) e (5) coincidono: e ciò è assurdo poíchè le $g_{n}(x)$ di $(4)$ non possono soddisfare alcun sistema del tipo (2).

Corollario. I'insieme dei punti singolari essenziali di una funzione analitica di due variabili complesse è perfetto $\left(^{*}\right)$. Infatti essso è chiuso: e per il teorema precedente non contiene alcun punto isolato.

5. Il teorema precedente si può immediatamente estendere quando al punto $(00)$ si sostituisca un punto $(\zeta n)$ generico, alla famiglia dei piani caratteristici $x=$ cost., una famiglia di varieta caratteristiche regolare nell'intorno del punto $(\xi, n)$. Con quest'ultima espressione vogliamo indicare una famiglia di superficie caratteristiche regolari nellintono di $(\xi)$ dipendenti analiticamente da un parametro a per modo che per ogni punto dell'intorno passi una ed una sola superficie della famiglia. Perchè l'equazione $\varphi(x, y, \alpha)$ rappresenti una tale famiglia occorre quindi e basta: $10^{\circ}$ che per $\alpha=\alpha_{0}$ sia $\varphi\left(\zeta, x_{0}\right)=0 ; 2 .^{\circ}$ che per ogui punto $(x y)$ dell'intoruo di $\left(\xi x_{1}\right)$ passi una ed una sola superficie della famiglia, in altri termini che l'equazione $\varphi(x, y, \alpha)=0$ si possa risolvere rapporto ad $\alpha$, e ciò̀ che sia $\left(\frac{\partial \psi}{\partial \alpha}\right)_{\left(\xi, \eta, \alpha_{0}\right)}=0 ; 3 .^{0}$ che $(\xi n)$ ed ogni punto dell'intorno sia regolare per la superficie passante per esso,

(*) Era noto che un punto singolare non pnò essere isolato, e quindi che l'insieme dei punti singolari è perfetto. Il corollario ci dice che esso rimane perfetto se ne sopprimiamo l'insieme dei punti singolari non essenziali. 
e cioè che una almeno delle variabili $x$ oppure $y$ si possa esprimere per una serie di potenze intere di $y$ od $x$ rispettivamente; deve esser quindi $\left(\frac{\partial \varphi}{\partial x}\right)_{\left(\xi, \eta, \alpha_{0}\right)}=\mid=0$, oppure $\left(\frac{\partial \varphi}{\partial y}\right)_{\left(\xi, \eta, \alpha_{3}\right)}=1=0$.

Ciò posto, a dimostrare la nostra affermazione, si scriva l'equazione della famiglia regolare di superficie nella forma $\psi(x y)=x$, il che per $2^{\circ}$ è possibile: e sia ad es. : $\left(\frac{\partial \psi}{\partial x}\right)_{(\xi \eta \eta)}=1=0$. Si faccia la trasformazione di variabili $x^{\prime}=\psi(x y)-\alpha_{0}$, $y^{\prime}=y-n ;$ poichè ̀̀ $\left(\frac{\partial \psi}{\partial x}\right)_{(\xi \eta)}=0$, ̀̀ questa una trasformazione regolare nel punto $\left(\xi \eta_{1}\right)$. Una funzione $f(x y)$ che abbia in $(\xi, n)$ una singolarità essenziale e che sia meromorfa nei punti della superficie $\downarrow(x y)=\alpha_{0}$ diversi da $(\xi, n)$, diviene una funzione $F\left(x^{\prime} y^{\prime}\right)$ avente una singolarità essenziale in $(0,0)$ e meromorfa negli altri punti di $x^{\prime}=0$. Essa avrà quindi nell'intorno di $x^{\prime}=0$ $y^{\prime}=0$ un punto singolare essenziale almeno su ogni piano $x^{\prime}=$ cost.; e cioè la $f(x y)$ avrà un punto singolare essenziale almeno nell'intorno di $\left(\zeta, x_{i}\right)$ su ogni superficie $\psi(x y)=\alpha$. Conchiuderemo dunque col seguente

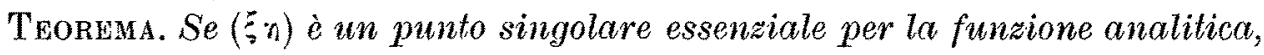
monodroma $f(x y)$; e si ha una famiglia regolare analitica di superficie caratteristiche nell'intorno di $(\xi n)$ tale che in questo intorno sulla superficie passante per $(\xi n)$, la $f(x y)$ è meromorfa tranne che nel punto $\left(\xi_{\circ}\right)$, sopra ogni superficie della famiglia esiste nell'intorno di $\left(\xi \pi_{1}\right)$ un punto singolare essenziale almeno di $f(x y)$.

6. Sarà questo il teorema fondamentale per le considerazioni cle seguono. Poichè ogni volta che, dato un insieme di punti, si può prendere una famiglia analitica di superficie caratteristiche regolare in un punto $(\sigma n)$ dell'insieme, tale che una di esse contenga il solo punto $\left(\zeta r_{i}\right)$ dell'insieme, ma infinite ne esistono che non ne contengono alcuno, potremo conchiudere che l'insieme dato non può essere l'insieme dei punti singolari essenziali di una funzione analitica.

$\mathrm{Ci}$ verrà sovente utile la seguente osservazione: $S e \varphi(x y)=0$ è una superficie caratteristica regolare in $\left(\xi r_{i}\right)$, la famiglia che se ne ottiene assoggettandola a tutte le trasiazioni che portano $(\xi, n)$ nei varii punti di un piano

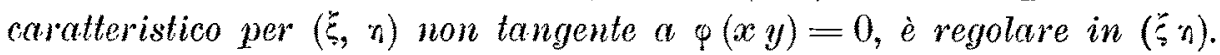

Infatti se $X-\xi-p(Y-n)=0$ è un tal piano, la famiglia d̀ data da $\varphi(x+p \alpha, y+x)=0$ : e si ha: 
1. ${ }^{\circ}$ per $\alpha=0, \varphi(\xi n)=0$;

2.0 poichè $\left(\frac{\partial \varphi}{\partial \alpha}\right)_{(\xi, \eta, 0)}=\left(\frac{\partial \varphi}{\partial x}\right)_{\langle\xi, \eta, 0)} p+\left(\frac{\partial \varphi}{\partial y}\right)_{(\xi, \eta, 0)}$ ed il piano $X-\xi=p\left(Y-n_{1}\right)$ non è tangente a $\varphi(x y)=0$ sarà certamente $\left(\frac{\partial \varphi}{\partial \alpha}\right)_{(\xi, \eta, 0)}=1=0$.

$3 .^{0}$ poichè $\varphi(x y)=0$ è regolare in $(\xi, n)$, è $\left(\frac{\partial \varphi}{\partial x}\right)_{(\xi, \eta, 0)}-1=0$ oppure è $\left(\frac{\partial \varphi}{\partial y}\right)_{(\xi, \eta, 0)}=\mid=0$

\section{§II.}

7. Sia E un insieme perfelto di punti, O un punto arbitrario fisso dello spazio, $r(O P)$ la distanza di $O$ dal punto generico $P$ di E. Se esiste un punto $P^{\prime}$ di $E$ tale che $r\left(O P^{\prime}\right)$ sia il massimo dei valori che $r(O P)$ prende nell' $n$ torno di $P^{\prime}$, non può esistere una funzione analitica nonodroma la quale abbia l'insieme $E$ come insieme di punti singolari essenziali.

Si supponga per semplicità che $O$ sia l'origine, che le coordinate di $P^{\prime}$ siano $\left(\xi, n_{1}\right)$ e che ad es. sia $\xi=\mid=0$.

Osserviamo anzitutto che è sempre possibile costruire una superficie caratteristica data da un'equazione

$$
x=\xi+a(y-n)+b(y-n)^{2}+c(y-n)^{3}+\cdots
$$

passante per $P^{\prime}$, regolare in questo punto e tale che i suoi punti nell'intorno di $P^{\prime}$ diversi da $P^{\prime}$ abbiano da $O$ una distanza maggiore (non mai uguale) di $r\left(O P^{\prime}\right)$ : basta perciò disporre convenientemente dei valori di $a$ e $b$. Invero, posto $a=a_{1}+i a_{2}, b=b_{1}+i b_{2}$, le equazioni reali contenute in (1) sono

$$
\left.\begin{array}{rl}
x_{1}-\xi_{1}=a_{1}\left(y_{1}-n_{1}\right) & -a_{2}\left(y_{2}-n_{2}\right)+b_{1}\left(y_{1}-n_{1}\right)^{2}- \\
& -b_{1}\left(y_{2}-n_{2}\right)^{2}+2 b_{2}\left(y_{1}-n_{1}\right)\left(y_{2}-n_{2}\right)+\cdots \\
x_{2}-\xi_{2}=a_{2}\left(y_{1}-n_{1}\right) & +a_{1}\left(y_{2}-n_{2}\right)+b_{2}\left(y_{1}-n_{1}\right)^{2}- \\
& \quad-b_{2}\left(y_{2}-n_{2}\right)^{2}+2 b_{1}\left(y_{1}-n_{1}\right)\left(y_{2}-n_{2}\right)+\cdots
\end{array}\right\}
$$

Quindi posto $\varphi\left(y_{1} y_{2}\right)=\frac{1}{2}\left(x_{1}^{2}+x_{2}^{2}+y_{1}^{2}+y_{2}^{2}\right)=\frac{1}{2} r^{2}(O P)$ dove $x_{1}, x_{2}$ 
sono date dalle (2) sarà :

$$
\begin{array}{cc}
\left(\frac{\partial \varphi}{\partial y_{1}}\right)_{(\xi \eta)}=\xi_{1} a_{1}+\xi_{2} a_{2}+n_{1} & \left(\frac{\partial \varphi}{\partial y_{2}}\right)_{(\xi \eta)}=\xi_{2} a_{1}-\xi_{1} a_{2}+n_{2} \\
\left(\frac{\partial^{2} \varphi}{\partial y_{1}^{2}}\right)_{(\xi \eta)}=1+|a|^{2}+2\left(\xi_{1} b_{1}+\xi_{2} b_{2}\right)\left(\frac{\partial^{2}}{\partial y_{2}^{2}}\right)_{(\xi \eta)}=1+|a|^{2}-2\left(\xi_{1} b_{1}+\xi_{2} b_{2}\right) \\
\left(\frac{\partial^{2} \varphi}{\partial y_{1} \partial y_{2}}\right)_{(\xi \eta)}=2\left(\xi_{2} b_{1}-\xi_{1} b_{2}\right) .
\end{array}
$$

Affinchè dunque la e quindi la distanza da $O$ dei punti di (1) abbia un minimo effettivo nel punto $\left(\xi, x_{1}\right)$ basterà che $: 10^{\circ}$ le $a_{1} a_{2}$ siano determinate per modo che

$$
\left.\begin{array}{l}
\left(\frac{\partial \varphi}{\partial y_{1}}\right)_{(\xi \eta)} \equiv \xi_{1} a_{1}+\xi_{2} a_{2}+n_{1}=0 \\
\left(\frac{\partial \varphi}{\partial y_{2}}\right)_{(\xi \eta)} \equiv \xi_{2} a_{1}-\xi_{1} a_{2}+n_{2}=0
\end{array}\right\}
$$

il che è sempre possibile poichè il determinante di queste equazioni lineari è uguale a $-|\xi|^{2}=1-0$ per ipotesi $; 2 .^{\circ}$ inoltre $b_{1}$ e $b_{2}$ siano tali che

$$
0>\left[\left(\frac{\partial^{2} \varphi}{\partial y_{1} \partial y_{2}}\right)^{2}-\frac{\partial^{2} \varphi}{\partial y_{1}^{2}} \frac{\partial^{2} \varphi}{\partial y_{2}^{2}}\right]_{(\xi \eta)} \equiv 4|\xi|^{2} \cdot|b|^{2}-\left(1+|a|^{2}\right)^{2}
$$

e

$$
\left(\frac{\partial^{2} \varphi}{\partial y_{1}^{2}}\right)_{(\xi \eta)}>0
$$

Ma quanto a (5) basterà prendere $|b|<\frac{1+|a|^{2}}{2|\xi|^{2}}$ perchè risulti soddisfatta; ed allora la (6) sarà soddisfatta di necessità poichè $\left(\frac{\partial^{2} \varphi}{\partial y_{1}^{2}}\right)_{(\xi \eta)}$ e $\left(\frac{\partial^{2} \varphi}{\partial y_{2}^{2}}\right)_{(\xi \eta)}$ avranno certamente il medesimo segno, e precisamente lo stesso segno della loro somma, che per le (3) è

$$
\left(\frac{\partial^{2} \varphi}{\partial y_{1}^{2}}\right)_{(\xi \eta)}+\left(\frac{\partial^{2} \varphi}{\partial y_{2}^{2}}\right)_{(\xi \eta)}=1+|a|^{2}>0
$$

Ciò posto, supponiamo dato un insieme $E$ quale è stato detto nell'enunciato. Costruiamo come si ̀̀ fatto ora una superficie caratteristica $S$ passante per $P^{\prime}$, regolare in $P^{\prime}$, tale che tutti i suoi punti nell'intorno di $P^{\prime}$ abbiano 
da $O$ una distanza maggiore di $r\left(O P^{\prime}\right)$ ed assoggettiamo $S$ a tutte le traslazioni che portano $P^{\prime}$ in un punto del piano caratteristico passante per $O P^{\prime}$. Questo piano noh può essere il piano tangente in $\left(\begin{array}{l}\xi \\ 3\end{array}\right)$ a $S$; infatti le (4) esprimono precisamente che il piano caratteristico tangente a $S$ giace nell'iperpiano $\xi_{1}\left(X_{1}-\xi_{1}\right)+\xi_{2}\left(X_{2}-\xi_{2}\right)+n_{1}\left(Y_{1}-n_{1}\right)+n_{2}\left(Y_{2}-n_{2}\right)=0$, normale alla retta che congiunge $O$ con $P^{\prime}$, la quale è invece contenuta nel piano caratteristico $O P^{\prime}$. Quindi pel n. 6 la famiglia di superficie caratteristiche così costruita è regolare nel punto $P^{\prime}$. E delle superficie di questa famiglia, la superficie $S$ ha nell'intorno di $P^{\prime}$ il solo punto $P^{\prime}$ comune con $E$; mentre le infinite superficie $S^{\prime}$ che si hanno per una traslazione sufficientemente piccola lungo la retta $\vec{O} \vec{P}^{\prime}$ nel verso da $O$ a $P^{\prime}$, non hanno nell'intormo di $P^{\prime}$ alcun punto comune con $E\left(^{*}\right)$. Se ora fosse $E$ l'insieme dei punti singolari essenziali di una funzione $f(x y)$ meromorfa e monodroma, la superficie $S$ non avrebbe nell'intorno di $P^{\prime}$ che il solo punto $P^{\prime}$ singolare essenziale per $f(x y)$, e le superficie $S^{\prime}$ non arrebbero punti singolari essenziali per $f(x y)$ : ora questo contraddice al teorema del 11.5 : quindi è assurda l'esistenza di $f(x y)$.

Corollarii. I. L'insieme dei punti singolari di una funzione $f(x y)$ non può contenere una parte perfetta isolata tutta al finito. Poichè evidentemente in una tale parte esisterebbe un punto quale il punto $P^{\prime}$ del teorema precedente.

In particolare si può dare a questo corollario la forma seguente:

II. Se una funzione $f(x y)$ è meromorfa in tutti $i$ punti di una ipersuperficie chiusa, è meromorfa all'interno di essa (**). Onde segue ad esempio che una funzione monodroma, meromorfa di due vaviabili non può avere spazii lacunari tutti al finito. E questo un primo teorema che contraddice all'affermazione del WeIERSTRAss richiamata al n. 1: ne vedremo tosto altri anche più interessanti nei nn. seguenti e nel $\$$ III.

8. Dal precedente teorema è però agevole trarre conseguenze un po' più nascoste che i precedenti corollarii.

Consideriamo nei piani $y=$ cost. il cerchio $|x| \leq r$, nei piani $x=$ cost.

(*) Poichè per tale traslazione il punto $P^{\prime}$ si allontanerà da $O$ e lo stesso accadrà dei punti di un intorno di $P^{\prime}$.

$\left.{ }^{*}\right)$ Per le funzioni olomorie in tutti i punti di una ipersuperficie chiusa vedi Hartogs, 1. c., Münch. Sitz. Ber. 
un campo $B_{1}$, che supponiamo tutto interno al cerchio $|y| \leq r_{1}$, e chiamiamo $C_{1}$ il suo contorno. Supponiano che una funzione $f(x y)$ sia meromorfa:

a) in tutti i punti $(0, y)$ dove $y$ è in $B_{1}$ o su $C_{1}$,

b) in tutti $i$ punti $(x y)$ dove y è su $C_{1}$ e $|x|<r$;

allora potremo affermare che la funzione $f(x y)$ è meromorfa in tutti $i$ punti $(x y)$ dove $|x|<r$ ed $y$ è in $B_{1}$.

Se il teorema non fosse vero, esisterebbe un punto singolare essenziale $(\xi)$ per cui $|\xi|<r$ ed $n$ è in $B_{1}$ : poniamo $|\xi|=p$.

Facciamo allora la trasformazione $X=\frac{k}{x}$ dove $k=\frac{r_{1} r p}{\sqrt{r^{2}-p^{2}}} \cdot$ La funzione $f(x y)$ diverrà una funzione $F(X y)$; e siccome la funzione $f(x y)$ è meromorfa nei punti $(0, y)$ quando $y$ è in $B_{1}$ o su $C_{1}$, e quindi pure esiste un numero $\sigma$ tale che $f(x y)$ è meromorfa in tutti i punti $(x y)$ per cui $y$ è in $B_{1}$ o su $C_{1}$ e $|x| \leqslant \sigma$, anche la $F(X y)$ è meromorfa in tutti i punti $(X, y)$ per cui $|X| \geq \frac{k}{\sigma}$ e $y$ è in $B_{1}$ o su $C_{1}$; e poichè la funzione $f(x y)$ è meromorfa in tutti i punti $(x y)$ per cui $|x|<r$ ed $y$ è su $C_{1}$, la $F(X y)$ è pure meromorfa in tutti i punti $(X y)$ per cui $|X|>\frac{k}{r}=\frac{r_{1} \rho}{\sqrt{r^{2}-\rho^{2}}}$ e $y$ su $C_{1}$; ed infine $F(X y)$ avrà nel punto $\left(\frac{k}{\xi}, n\right)$, per l'ipotesi fatta su $f(x y)$, un punto singolare essenziale interno al campo $\left[|X|>\frac{r_{1} \rho}{\sqrt{r^{2}--\rho^{2}}}, y\right.$ in $\left.B_{1}\right]$.

Consideriamo l'insieme $E_{1}$ dei punti singolari essenziali di $F(X y)$ all'interno o sul contorno del campo $\left[|X|>\frac{r_{1} p}{\sqrt{r^{2}-p^{2}}}, y\right.$ in $B_{1}$ o su $\left.C_{1}\right]$; sarà questo un insieme perfetto tutto al finito, e di più, poichè la funzione $F(X y)$ è meromorfa per $y$ su $C_{1}$ ed $|X|>\frac{r_{1} \rho}{\sqrt{r^{2}-\rho^{3}}}$, se esistono punti di $E_{1}$ sul contorno del campo essi appartengono necessariamente alla varietà $|X|=\frac{r_{1} p}{\sqrt{r^{2}-p^{2}}}, y$ in $B_{1}$. D'altra parte se diciamo $R$ il massimo della distanza di un punto di $E_{1}$ dall'origine, poichè $\left(\frac{k}{\xi}, n\right)$ è un punto di $E_{1}$ sarà certamente $R \supseteq \sqrt{\frac{k^{2}}{\rho^{2}}+|n|^{2}} \geq \frac{k}{\rho}=\frac{r r_{1}}{\sqrt{r^{2}-p^{2}}} \cdot$ Ne segue che un punto $P^{\prime}$ di $E_{1}$ il quale disti dall'origine di $R$ è certamente interno al campo $\left[|X|>\frac{r_{1} \rho}{\sqrt{r^{2}-\rho^{2}}}\right.$, 
$y$ in $B_{1} \quad 0$ suc $\left.C_{1}\right]$, perchè la sfera $|X|^{2}+|y|^{2}=R^{2}$ incontra la varietà $|X|=\frac{r_{1} \rho}{\sqrt{r^{2}-\rho^{2}}}$ in punti per cui è $|y|=\sqrt{R^{2}-\frac{r_{1}^{2} \rho^{2}}{r^{2}-\rho^{2}}}=r_{1}$ e quindi certo all'esterno del campo $B_{i}$.

Trovandosi $P^{\prime}$ all' interno - e non sul contorno - del campo $\left[|X|>\frac{r_{1} \rho}{\sqrt{r^{2}-p^{2}}}, y\right.$ inl $\left.B_{1}\right]$, ne segue che nell'intorno di $P^{\prime}$ non si trovano altri punti singolari essenziali di $F(X y)$ oltre a quelli di $E_{1}$; onde il punto $P^{\prime}$ si troverà nelle condizioni del teorema del n. precedente: il che è assurdo. Quindi anche $f(x y)$ non può certamente avere un punto singolare essenziale all'interno del campo $|x|<r, y$ in $B_{1}$ c. v. $d$.

9. Invece di considerare sui piani $y=$ cost. il cerchio $|x|=r$, si potrà evidentemente considerare un campo $B$ che si possa rappresentare conformemente su un cerchio di raggio $r$ : poichè se $x^{\prime}=\psi(x)$ è la funzione analitica della variabile $x$ che dà questa rappresentazione conforme, la trasformazione $x^{\prime}=\psi(x), y=y$ sarà regolare per $x$ interuo a $B$ ed $y$ qualunque, onde muta la funzione $f(x y)$ in una funzione $F\left(x^{\prime} y\right)$ che sarà meromorfa all'interno del campo $\left[\left|x^{\prime}\right|<r, y\right.$ in 3$]$, allora ed allora soltanto che la funzione $f(x y)$ è meromorfa per $x$ interno al campo $B$ ed $y$ interno al. campo $B_{1}$. Onde potremo conchiudere:

Se $B$ rappresenta un campo qualunque nei piani $y=$ cost. che si possa rappresentare conformemente su un cerchio ed $x=a$ è un punto di esso, se $B_{1}$ rappresenta un campo interno al cerchio $|y| \leq v_{1}$ e $C_{1}$ ne indica il contorno, se infine $f(x y)$ è una funzione meromorfa

a) in tutti $i$ punti $(\alpha y)$ dove $y$ è in $B_{1}$ o su $C_{1}$,

b) in tutti i punti (xy) dove $x$ è in $B$ e y su $C_{1}$, allora potremo affermare che la funzione $f(x y)$ è meromorfa in tutti $i$ punti $(x y)$ per cui $x$ è in $B$ ed $y$ in $B_{1}$ o su $C_{1}(*)$.

10. Sia $f(x y)$ una funzione meromorfa sul piano $x=0$ quando $y$ è interno ad un certo campo $B_{1}$ tutto al finito o sul contorno $C_{1}$ di esso; sopra ogni piano $y=y_{0}, y_{0}$ essendo un punto di $B_{1} \circ C_{1}$, esisterà un massimo

(*) Pel caso che la $f(x y)$ sia olomorfa vedi Hartogs, 1. c. $2 .^{\circ}$

Annali ai Matematica, Serie III, Tomo XVII. 
cerchio di centro $x=0$ entro cui $f(x y)$ è meromorfa. Diciamo $R_{y_{0}}$ il raggio di tale cerchio (*): sarà $R_{y_{0}}$ la minima distanza dal piano $x=0$ dei punti singolari essenziali di $f(x y)$ appartenenti al piano $y=y_{0}$. Osserviamo subito per la $R_{y}$ la seguente proprietà :

La funzione $R_{y}$ è semicontinua inferiormente; ossia, assegnato un numero s, è sempre possibile trovare un numero $\delta$ tale che per $\left|y-y_{0}\right|<\delta$ sia $R_{y} \supseteq R_{y_{0}}-\varepsilon$.

Infatti ove ciò non fosse esisterebbero infiniti valori $y_{1}, y_{2}, \ldots, y_{i}, \ldots$ tali che $\lim _{i=\infty} y_{i}=y_{0}$ e $R_{y_{i}}<R_{y_{0}}-\varepsilon$. I punti singolari essenziali $\left(x_{i} y_{i}\right)$ con $\left|x_{i}\right|=R_{i}$ che si trovano nei piani $y=y_{i}$ avrebbero allora un punto limite $\left(x_{0} y_{0}\right)$ con $\left|x_{0}\right| \leq R_{y_{0}}$. $-\varepsilon$, e questo sarebbe necessariamente un punto singolare essenziale, il che è assurdo poichè per ipotesi entro al cerchio $|x|<K_{3, \mathrm{~h}}$ sul piano $y=y_{0}$ non esistono punti singolari essenziali.

Ma dal teorema del n. 8 segue una proprietà ancora più notevole:

Se $p_{y}$ è una funzione reale di $y_{1}$ ed $y_{2}$, definita in $B_{1}$, che sul contorno $C_{1}$ soddisfa alla limitazione $0<p_{y} \leq R_{y}$ ed all' interno di $B_{1}$ all' equazione $\Delta^{*}{ }_{2} \log p_{y}=0\left({ }^{* *}\right)$, in tutti $i$ punti di $B_{1}$ sarì pure

$$
p_{y} \leq R_{y} \text {. }
$$

Infatti su $C_{1}$, poichè è $0<p_{y} \leqslant R_{y}, \log p_{y}$ è sempre finito: l'ipotesi che in $B_{1}$ sia $\Delta^{\prime \prime}{ }_{2} \log p_{y}=0$ porta che $\log p_{y}$ sia sempre finita e regolare: quindi sarà sempre $p_{y}>0$ anche all'interno di $B_{1}$.

Indichiamo con $q_{y}$ la funzione coningata di $\log p_{y}$ - fissandone arbitrariamente la.costante additira -; le funzioni

$$
\begin{aligned}
& \varphi(y)=\log p_{y}+i q_{y} \\
& \psi(y)=e^{\varphi(y)}=p_{y} e^{i q_{y}}
\end{aligned}
$$

sono funzioni regolari analitiche di $y$ in $B_{1}$; e sarà $|\psi(y)|=p_{y}>0$.

(*) L'Hartoas chiama analogamente $R_{x,}^{*}$ la minima distanza di un punto singolare essenziale o no del piano $x=x_{0}$ dal piano $y=0$. Cfr. 1. c., Math. Ann. E mediante considerazioni sugli sviluppi in serie dimostra per questa funzione proprietà analoghe a quelle che seguono nel testo. Cfr. specialmente i $\$ \S 5,6,8,10$.

$\left(^{* *}\right)$ Poniamo qui e nel seguito $\Delta_{2}^{\prime}=\frac{\partial^{2}}{\partial x_{1}^{2}}+\frac{\partial^{2}}{\partial x_{2}^{2}}, \quad \Delta_{2}^{\prime \prime}=\frac{\partial^{2}}{\partial y_{1}^{2}}+\frac{\partial^{2}}{\partial y_{2}^{2}}$. 
Facciamo allora la trasformazione

$$
X=\frac{x}{\psi(y)} \quad Y=y
$$

È questa una trasformazione regolare invertibile in tutti i punti per cui $y$ è interno a $B_{1}$ o su $C_{1}$; e se, come segue dall'ipotesi che su $C_{1}$ sia $R_{y} \supseteq p_{y}$ la $f(x y)$ è meromorfa

a) nei punti $(0 y)$ per cui $y$ è in $B_{1}$ o su $C_{1}$,

b) nei punti $(x y)$ quando $y$ ̀̀ su $C_{1}$ e $|x|<p_{y}$;

la funzione $F(X Y)$ trasformata di $f(x y)$ per la (8) è meromorfa

$\left.a^{\prime}\right)$ nei punti $(0 Y)$ per cui $Y$ è in $B_{1}$ o su $C_{1}$,

$\left.b^{\prime}\right)$ nei punti $(X Y)$ per cui $Y$ è su $C_{1}$ e $|X|<1$.

Segue dal teorema del n. 8 che $F(X Y)$ è meromorfa in tutti i punti $(X Y)$ per cui $Y$ è in $B_{1}$ o su $C_{1}$, e $|X|<1$; ossia che $f(x y)$ è meromorfa in tutti i punti per cui $y$ è in $B_{1}$ o su $C_{1}$, e $|x|<|\dot{\psi}(y)|=p_{y}$. Quindi sarà certo $R_{y} \supseteq p_{y}$ : c. v. d.

Dai due teoremi dimostrati per $R_{y}$ seguono, ragionando come fa l'Hak'Togs nella Memoria citata dei Math. Ann. al $\$ 8$ (u. 1, Zusatz, pag. 47 e 48 ; e n. 3), i seguenti corollarii :

1. Mantenute le notazioni del teorema precedente, se anche in un sol punto $y_{0}$ interno a $B_{1} \grave{e} R_{y_{0}}=p_{3 n}$ sarì allora ovunque in $B_{1}$

$$
R_{y}=p_{y}
$$

2. ${ }^{\circ}$ Se $R_{y}$ ammelle le derivate dei primi due ordini rapporto ad $y_{1}$ e $y_{2}$, essa soddisfa alla disuguaglianza

$$
\Delta^{\prime \prime} \log R_{y} \leq 0
$$

Il teorema precedente ed il suo primo corollario sono - insieme col teorema fondamentale del $\$$ I - i teoremi su cui fonda l'Hartogs tutti i ragionamenti che svolge nella Memoria citata degli Acta Mathematica: senza ripeterli quindi noi potremo conchiudere coi seguenti enunciati che si ottengono da quelli dell'Hartogs mutando le parole «funzione regolare » e «punto singolare, nelłe parole «funzione meromorfa $\mathrm{e}$ «punto singolare essenziale. »

I. Se $x=0 y=0$ è un punto singolare essenziale per una funzione ana- 
litica $f(x y)$ monodroma $\left({ }^{*}\right) d i x$ ed $y$; e se l'insieme dei punti singolari essenziali di $f(x y)$ in un certo intorno $|x|<\rho,|y|<\rho^{\prime}$ dell'origine $\grave{e}$ cos̀̀ costituito che in ogni piano $x=x_{0}$ di esso vi sia al piu un punto singolare essenziale mentre nei residui la funzione è meromorfa, esiste allora su ogni piano $x=\xi$ dove 5 appartiene ad un conveniente intorno dello zero un punto singolare essenziale $(\xi, \varphi(\xi))$, e la funzione $\varphi(\xi)$ è una funzione analitica regolare $d i \xi$ (l. c., pag. 70):

II. Se nelle stesse condizioni del teorema precedente su ogni piano $x=\xi=0$ esistono proprio $r$ putnti singolari essenziali di $f(x y) n_{1}, n_{2}, \ldots, n_{n}$, le loro funzioni simmetriche elementari sono funzioni regolari analitiche di $\xi$; od in altri termini le $n_{i}(\xi)$ sono funzioni analitiche di $\xi_{\zeta}^{\xi}$ le quali per $\xi=0$ hanno al pii un punto di diramazione (l. c., pag. 76).

Non tralasceremo di notare che la proprietà dimostrata per la funzione $R_{y}$ è in certa qual guisa caratteristica per essa: precisamente che se è assegnata una funzione $U_{y}$ reale, finita, avente derivate prime e seconde finite $e$ continue e soddisfacente alla condizione $\Delta^{\prime \prime}{ }_{2} U_{y}=0$ in un campo $T$, è sempre possibile trovare una funzione $f(x y)$ per cui la funzione $R_{y}$ sia in $T$ uguale $a d e^{U_{v}}$. Infatli in queste ipotesi l'HakTogs costruisce (**) una funzione $p(x y)$ che ha in ogni piano $y=y_{0}$ sopra il cerchio di raggio $e^{U_{y}}$ e centro l'origine un punto singolare essenziale o no, ed è regolare all'interno del cerchio: basterà allora considerare la funzione $f(x y)=e^{\varphi(x y)}$, perchè la nuova funzione ottenuta pure restando regolare all'interno di quei cerchi ahbia sul contorno di essi una singolarità essenziale.

\section{$\S$ III.}

11. Sia $\varphi\left(x_{1}, x_{2}, y_{1}, y_{2}\right)=0$ una ipersuperficie $S$ dello spazio a 4 dimensioni : vogliamo esaminare quando essa possa essere frontiera del campo di esistenza di una funzione analitica meromorfa: in altri termini vogliamo

$\left(^{*}\right)$ Evidentemente si può anche supporre $f(x y)$ un ramo monodromo vell'intorno del punto $(00)$ di una funzione polidroma. L'HARToGs tratta anche il caso in cui $f(x y)$ abbia un punto critico in $(00)$, non sarebbe difficile anche a noi raggiungere con qualche complemento la stessa estensione.

(**) L. c., Math. Ann., $\$ 10$. 
vedere quando la $S$ è tale che esiste una funzione analitica meromorfa in una, delle regioni. $n$ che hanno $S$ per frontiera per cui tutti $\mathrm{i}$ punti di $S$ siano singolari essenziali. Cambiando al più il segno di $\varphi$ possiamo supporre che la regione $\Pi$ sia quella in cui è

$$
\left(x_{1} x_{2} y_{1} y_{2}\right)>0
$$

Applicheremo ancora il teorema del $\S \mathrm{I}$. Consideriamo un punto $P \equiv\left(\zeta \pi_{1}\right)$ di $S$, e supponiamo di poter costruire una superficie caratteristica $\Sigma$ che passi per $P$, ed ivi sia regolare e tocchi l'ipersuperficie $S$, ed infine non abbia nell'intorno di $P$ altri punti comuni con $S$ : Essa cadrà nell'intorno di $P$ tutta in una delle due regioni in cui $S$ divide lo spazio: affinchè nella regione $\varphi>0$ possa esistere una funzione $f(x y)$ che ammetta $S$ come frontiera, è necessario che ogni tale superficie caratteristica cada nella regione $\varphi<0$.

Infatti assoggettiamo $\Sigma$ alle traslazioni che portano $P$ nei punti del piano caratteristico che contiene la normale a $S$ per $P$; poichè questo piano non è tangente a $\Sigma$, la famiglia di superficie così generata è una famiglia regolare in $P$. Inoltre le superficie corrispondenti ad una traslazione sufficientemente piccola diretta secondo la normale a $S$ in $P$ volta verso la regione in cui cade $\Sigma$ non hanno nell' intorno del punto. $P$ punti comuni con $S\left(^{*}\right)$. Se quindi $\Sigma$ giacesse nella regione $\varphi>0$, la $f(x y)$ sarebbe meromorfa in tutti i punti di $\Sigma$ dell'intorno di $P$, fuori che in $P$, e meromorfa in tutti i punti dell'intorno di $P$ di queste superficie: e ciò per il teorema I è assurdo.

12. Cerchiamo dunque di costruire una superficie caratteristica $\Sigma$ che goda rispetto a $S$ nel punto $\left(\zeta x_{1}\right)$ delle proprietà dette sopra. Supporremo che la $p$ abbia le derivate dei primi due ordini linite e continue. In un punto regolare di $S$ non si avrà $\frac{\partial \varphi}{\partial x_{1}}=\frac{\partial \varphi}{\partial x_{z}}=\frac{\partial \varphi}{\partial y_{1}}=\frac{\partial \varphi}{\partial y_{2}}=0 ;$ onde sarà

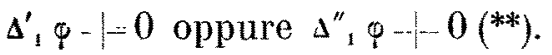

(*) Tale affermazione è geometricamente evidente: ci si può ridure all'intuizione geometrica nello spazio a 3 dimensioni segando con un inerpiano variabile attorno alla normale a $S$ in $P$.

(**) In modo analogo a quanto si fece al n. 10 poniamo

$$
\Delta_{1}^{\prime}=\left(\frac{\partial}{\partial x_{1}}\right)^{2}+\left(\frac{\partial}{\partial x_{2}}\right)^{2} ; \quad \Delta_{1}^{\prime \prime}=\left(\frac{\partial}{\partial y_{1}}\right)^{2}+\left(\frac{\partial}{\partial y_{2}}\right)^{2} ; \quad \Delta_{2}^{\prime}=\frac{\partial^{2}}{\partial x_{1}^{2}}+\frac{\partial^{2}}{\partial x_{2}^{2}} ; \quad \Delta_{2}^{\prime \prime}=\frac{\partial^{2}}{\partial y_{1}^{2}}+\frac{\partial^{2}}{\partial y_{2}^{2}} .
$$


Supporremo, per fissare le idee, che nel punto $\left(\xi r_{1}\right)$ sia $\Delta_{1}^{\prime} \varphi==0$.

La superficie $\Sigma$ sia allora data come nel $\S$ II dall'equazione tra le variabili complesse :

$$
x=\xi+a(y-n)+b(y-n)^{2}+c(y-n)^{3}+\cdots
$$

e cioè dal sistema di equazioni tra le variabili reali :

$$
\begin{aligned}
x_{1}-\xi_{1}=a_{1}\left(y_{1}-n_{1}\right) & -a_{2}\left(y_{2}-n_{2}\right)+b_{1}\left(y_{1}-n_{1}\right)^{2}- \\
& -b_{1}\left(y_{2}-n_{2}\right)^{2}-2 b_{2}\left(y_{1}-n_{1}\right)\left(y_{2}-n_{2}\right)+\cdots \\
x_{2}-\xi_{2}=a_{2}\left(y_{1}-n_{1}\right) & +a_{1}\left(y_{2}-n_{2}\right)+b_{2}\left(y_{1}-n_{1}\right)^{2}- \\
& -b_{2}\left(y_{2}-n_{2}\right)^{2}+2 b_{1}\left(y_{1}-n_{1}\right)\left(y_{2}-n_{12}\right)+\cdots
\end{aligned}
$$

Calcolando il valore di $\varphi\left(x_{1} x_{2} y_{1} y_{2}\right)$ nei punti di $\Sigma$ avremo dunque, ricordando che $P$ è su $S$ ossia che $\varphi\left(\xi_{1} \xi_{2} n_{1} n_{2}\right)=0$ :

$$
\begin{aligned}
\varphi\left(x_{1} x_{2} y_{1} y_{2}\right) & =\left(y_{1}-n_{1}\right)\left[\frac{\partial \varphi}{\partial x_{1}} a_{1}+\frac{\partial \varphi}{\partial x_{2}} a_{2}+\frac{\partial \varphi}{\partial y_{1}}\right]+ \\
& +\left(y_{2}-x_{2}\right)\left[\frac{\partial \varphi}{\partial x_{2}} a_{1}-\frac{\partial \varphi}{\partial x_{1}} a_{2}+\frac{\partial \varphi}{\partial y_{2}}\right]+ \\
& +\left(y_{1}-n_{1}\right)^{2}\left[\frac{\partial \varphi}{\partial x_{1}} b_{1}+\frac{\partial \varphi}{\partial x_{2}} b_{2}+A_{1}\right]+ \\
& +\left(y_{2}-n_{2}\right)^{2}\left[-\frac{\partial \varphi}{\partial x_{1}} b_{1}-\frac{\partial \varphi}{\partial x_{2}} b_{2}+C_{1}\right]+ \\
& +2\left(y_{1}-n_{1}\right)\left(y_{2}-x_{12}\right)\left[\frac{\partial \varphi}{\partial x_{2}} b_{1}-\frac{\partial \varphi}{\partial x_{1}} b_{2}+B_{1}\right]+\cdots
\end{aligned}
$$

i termini tralasciati essendo di ordine maggiore del secondo nelle quantità $y_{1}-n_{1}, y_{2}-r_{12}$. Nella (3) si deve intendere che le $\frac{\partial \varphi}{\partial x_{1}}, \frac{\partial \varphi}{\partial x_{2}}, \frac{\partial \psi}{\partial y_{1}}, \frac{\partial \varphi}{\partial y_{2}}$ siano calcolate nel punto $\left(n_{1}\right)$ : inoltre $A_{\mathfrak{q}}, B_{1}, C_{1}$ non dipendono da $b_{1}$ e $b_{2}$ e precisamente è :

$$
\begin{aligned}
& \mathcal{2} A_{1}=\frac{\partial^{2} \varphi}{\partial x_{1}^{2}} a_{1}^{2}+2 \frac{\partial^{2} \varphi}{\partial x_{1} \dot{\partial} x_{2}} a_{1} a_{2}+\frac{\partial^{2} \varphi}{\partial x_{2}^{2}} a_{2}^{2}+2 \frac{\partial^{2} \varphi}{\partial x_{1} \partial y_{1}} a_{1}+2 \frac{\lambda^{2} \varphi}{\partial x_{2} \dot{\partial} y_{1}} a_{2}+\frac{\partial^{2} \varphi}{\partial y_{1}^{2}}, \\
& B_{1}=-\left(\frac{\partial^{2} \varphi}{\partial x_{1}^{2}}-\frac{\partial^{2} \varphi}{\partial x_{2}^{2}}\right) a_{1} a_{2}+\frac{\partial^{2} \varphi}{\partial x_{1} \partial x_{2}}\left(a_{1}^{2}-a_{2}^{2}\right)+\left(\frac{\partial^{2} \varphi}{\partial x_{1} \partial y_{2}}+\frac{\partial^{2} \varphi}{\partial x_{2} \partial y_{1}}\right) a_{1}- \\
& -\left(\frac{\partial^{2} \varphi}{\partial x_{1}} \frac{\varphi}{\partial y_{1}}-\frac{\partial^{2} \varphi}{\partial x_{2}} \hat{\partial} y_{2}\right) a_{2}+\frac{\partial^{2} \varphi}{\partial y_{1}} \frac{\partial}{\partial y_{2}},
\end{aligned}
$$

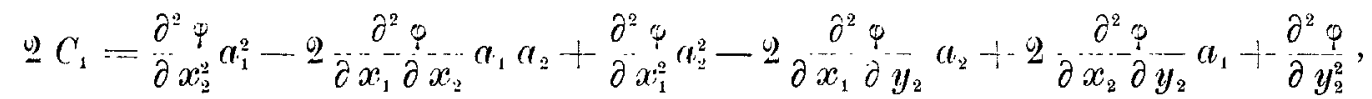


dove però le derivate seconde debbono intendersi tutte calcolate in un punto intermedio del segmento che unisce $\left(\xi_{1}, \xi_{2}, x_{1}, x_{2}\right)$ con $\left(x_{1}, x_{2}, y_{1}, y_{2}\right)$. Affiuchè la superficie $\Sigma$ data da (2) abbia il solo punto $P$ comune con $S$ occorrerà intanto che sia

$$
\frac{\partial \varphi}{\partial x_{1}} a_{1}+\frac{\partial \varphi}{\partial x_{2}} a_{2}+\frac{\partial \varphi}{\partial y_{1}}=0, \frac{\partial \varphi}{\partial x_{2}} a_{1}-\frac{\partial \varphi}{\partial x_{1}} a_{2}+\frac{\partial \varphi}{\partial y_{2}}=0 .
$$

Per l'ipotesi che in $(\xi n)$ sia $\Delta_{1}^{\prime} \varphi=0$ queste equazioni ammettono sempre soluzione; e precisamente si ha

$$
\begin{aligned}
& \left.a_{1}=-\frac{1}{\Delta_{1}^{\prime} \varphi} \mid \frac{\partial \varphi}{\partial x_{1}} \frac{\partial \varphi}{\partial y_{1}}+\frac{\partial \varphi}{\partial x_{2}} \frac{\partial \varphi}{\partial y_{2}}\right\} \mid \\
& \boldsymbol{a}_{2}=\frac{1}{\boldsymbol{\Delta}_{1}^{\prime} \varphi}\left|\frac{\partial \varphi}{\partial \boldsymbol{x}_{1}} \frac{\partial \varphi}{\partial y_{2}}-\frac{\partial \varphi}{\partial x_{2}} \frac{\partial \varphi}{\partial y_{1}}\right| \mid
\end{aligned}
$$

Affinchè $\Sigma$ sia dunque una delle superficie richieste occorre cle $a_{1}$ ed $a_{2}$ slano dati dalle (6); riconosciamo subito con cio che necessariamente è anche soddisfatta la condizione che $\Sigma$ ed $S$ siano tangenti poichè le (5) esprimono precisamente che il piano tangente a $\mathbf{x}$ giace nelliperpiano tangente a $S$.

Ma le condizioni precedenti non bastano ad assicurare che $\Sigma$ abbia il solo punto $P$ comune con $S$; perciò basterà invece determinare $b_{1}$ e $b_{2}$ per modo che la forma quadratica costituita dai termini di secondo grado di (3) sia definita: ed anzi per la supposta continuità delle derivate seconde di $\varphi$, basterà che sia definita quella forma che si ottiene da questa col prendere per le derivate seconde delle $\varphi$ che compaiono nei coefficienti di quella forma $i$ valori che esse assumono nel punto $\left(\xi_{n}\right)$; - e così intenderemo di fare d'ora in poi -. Ed allora affinchè $\Sigma$ giaccia nel campo $\varphi<0$ occorrerà che la forma sia definita negativa.

Ora affinchè questa forma sia definita occorre si abbia

$\left(\frac{\partial \varphi}{\partial x_{2}} b_{1}-\frac{\partial \varphi}{\partial x_{1}} b_{2}+B_{1}\right)^{2}+\left(\frac{\partial \varphi}{\partial x_{1}} b_{1}+\frac{\partial \varphi}{\partial x_{2}} b_{2}+A_{1}\right)\left(\frac{\partial \varphi}{\partial x_{1}} b_{1}+\frac{\partial \varphi}{\partial x_{2}} b_{2}-C_{1}\right)<0$

ed allora essa sarà definita positiva o negativa a seconda che ̀̀ $>0$ oppure $<0$ la somma dei coefficienti di $\left(y_{1}-n_{1}\right)^{2}$ e $\left(y_{2}-n_{2}\right)^{2}$; ossia secondo che

$$
A_{1}+C_{1}>0 \text { oppure } A_{1}+C_{1}<0 \text {. }
$$

Ma perchè $b_{1}$ e $b_{2}$ si possano determinare per modo che (7) sia soddisfatta occorre e basta che sia $A_{1}+C_{1}=0$ : perchè se $A_{1}+C_{1}=0$ basta 
determinare $b_{1}$ e $b_{2}$ colle condizioni che $\frac{\partial \varphi}{\partial x_{2}} b_{1}-\frac{\partial \varphi}{\partial x_{1}} b_{2}+B_{1}=0$ e che $\frac{\partial \varphi}{\partial x_{1}} b_{1}+\frac{\partial \varphi}{\partial x_{2}} b_{2}$ sia compreso fra $-A_{1}$ e $C_{1}$ perchè risulti soddisfatta la (7), mentre se $A_{1}=-C_{1}$ il primo membro di (7), come somma di due quadrati, risulterà $\geq 0$.

Concludiamo dunque che se $A_{1}+C_{1}>0$ si può rendere la nostra forma definita positiva, se $A_{1}+C_{1}<0$, la si può rendere definita negativa. E quindi infine che affinchè non si possa costruire una superficie $\Sigma$ come quella indicata al n. precedente, oppure che essa cada necessariamente nella regione $\varphi<0$, occorre sia

$$
A_{1}+C_{1} \leq 0 \text {. }
$$

Sostituiamo ad $A_{1}, C_{1}, a_{1}, a_{2}$ i valori dati da (4) e (6): la (8) diviene

$$
\begin{aligned}
A_{1}+C_{1}=\frac{1}{2 \Delta_{1}^{\prime} \varphi} \mid \Delta_{2}^{\prime} \varphi \cdot \Delta^{\prime \prime}{ }_{1} \varphi+\Delta_{2}^{\prime \prime}{ }_{2} \rho \cdot \Delta_{1}^{\prime} \varphi-2 & {\left[\frac{\partial \varphi}{\partial x_{1}} \frac{\partial \varphi}{\partial y_{1}}+\frac{\partial \varphi}{\partial x_{2}} \frac{\partial \varphi}{\partial y_{2}}\right]\left[\frac{\partial^{2} \varphi}{\partial x_{1} \partial y_{1}}+\frac{\partial^{2} \varphi}{\partial x_{2} \partial y_{2}}\right]-} \\
& -2\left[\frac{\partial \varphi}{\partial x_{1}} \frac{\partial \varphi}{\partial y_{2}}-\frac{\partial \varphi}{\partial x_{2}} \frac{\partial \varphi}{\partial y_{1}}\right]\left[\frac{\partial^{2} \varphi}{\partial x_{1} \partial y_{2}}-\frac{\partial^{2} \varphi}{\partial x_{2} \partial y_{1}}\right] \mid \leq 0
\end{aligned}
$$

Poichè si è supposto $\Delta_{1}^{\prime} \varphi==0$ e quindi $>0$, la (9) si tradurrà quindi nel chiedere che la parte tra parentesi del secondo membro di (9) sia $\leq 0$. In tale forma la condizione (9) viene ad essere simmetrica nelle due coppie di variabili $x_{1}, x_{2}$ ed $y_{1}, y_{2}$, onde essa risulta indipendente dalla ipotesi che sia $\Delta_{1}^{\prime} \varphi=-0$ piuttosto che $\Delta^{\prime \prime}{ }_{1} \varphi=1=0$.

Raccogliendo:

Affinchè una ipersuperficie $S$ di equazione p $\left(x_{1} x_{2} y_{1} y_{2}\right)=0$ possa essere frontiera per una funzione $f(x y)$ analitica, nonodroma, meromorfa in uno dei campi in cui essa divide lo spazio (od in parte di esso) è necessario che la quantità

$$
\begin{aligned}
& \mathfrak{S} \varphi \equiv \Delta_{2}^{\prime} \phi \cdot \Delta^{\prime \prime}{ }_{1} p+\Delta^{\prime \prime}{ }_{2} \varphi \cdot \Delta^{\prime}{ }_{1} p-2\left|\frac{\partial \varphi}{\partial x_{1}} \frac{\partial \varphi}{\partial y_{1}}+\frac{\partial p}{\partial x_{2}} \frac{\partial \varphi}{\partial y_{2}}\right|\left|\frac{\partial^{2} \varphi}{\partial x_{1} \partial y_{1}}+\frac{\partial^{2} \varphi}{\partial x_{2} \partial y_{2}}\right|-\mid
\end{aligned}
$$

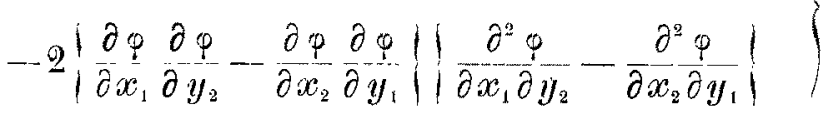

conservi nei punti di $S$ un segno determinato. 
Se si ha sempre $\mathfrak{d} \varphi \leq 0$ una funzione $f(x y)$ che abbia $S$ come frontiera può esistere solo nel campo in cui $\grave{e} y>0$; se si hic sempre $\mathfrak{d} q \geq 0$ ma funzione $f(x y)$ che abbia $S$ per frontiera può esistere solo nel campo $\varphi<0$.

Corollario. Le sole ipersuperficie $\because=0$ che possono essere frontiern comme per due fumzioni f $(x y)$ meromorfe l'ma nel campo $i>0$, l'altra nel campo $\because<0$ sono le soluzioni dell equazione differenziale

$$
\mathfrak{Q} \mathfrak{q}=0 \text {. }
$$

13. Sarebbe ora assai interessante rispondere alla domancia: Le condizioni precedtenti sono caratteristiche per le ipersuperficie che possono esseve frontiera di qualche funzione analitica? Con maggior precisione: data ma funzione $\tau_{i}\left(x_{1} x_{2} y_{1} y_{2}\right)=0$ tale che nei pmati dello ipersuperficie $\varphi_{1}=0$ (o di $\mathrm{mm}$ pezzo di essa) sia sempre $\mathfrak{E} y \geq 0$, esiste sempre nna funzione $f(x y)$ analitica, monodroma, meromorfa nei punti del campo $\psi<0$ dell'intomo della ipersuperficie, che abbia l'ipersuperficie medesima (o quella parte di essa) come frontiera e cioè come varietì di munti singolari essenziali.

Non mi è riuscilo di dare a questa domanda ma risposta esauriente; mi pare tutlaria the non sia privo di interesse il mostrare come net caso particolare in cui la funzione op $\left(x_{1} x_{2} y_{1} y_{3}\right)$ soddisfacca nei muti in cui $\eta_{1}=0$ all'equarione

$$
\mathfrak{d} \phi=0
$$

è realmente possibile costrurve funzioni meromorfe sia nel campo $q<0$ che nel crmpo $\%>0$ che abbiano per frontiera l'ipersuperficie $S$ od alimeno una parte di essa sufficientemente limitata: risultato che mi pare renda molto probabile che anche alla domanda più generale fatta sopra si dovrà rispondere affermativamente.

Dimostreremo perciò il seguente lemma che m pare anche interessante per se medesimo :

Le ipersuperficie $\left(x_{1} x_{2} y_{1} y_{2}\right)=0$ tali che nei loro punti la funzione soddisfa l'equazione (11) sono tutte e sole le ipersuperficie dello spazio a quattro aimensioni composte con una semplice infiniti di superficie caratteristiche.

Affinchè l'jpersuperficie

$$
\vartheta\left(x_{1} x_{2} y_{1} y_{2}\right)=0
$$

sia composta con $\boldsymbol{\alpha}^{1}$ superficie caratteristiche è necessario e sufficiente che si possa trovare una funzione $\psi\left(x_{1} x_{2} y_{1} y_{2}\right)$ tale che associando alla (19) l'eAnnali di Matematica, Serie III, Tomo XVII. 
quazione

$$
\psi\left(x_{1}, x_{2}, y_{2}\right)=\alpha
$$

dove $x$ indica un parametro arbitrario, si possano dalle (12) e (13) ricavare ad es.: le $x_{1}, x_{2}$ in funzione di $y_{1} y_{2}$ in molo che risultino soddisfatte le equazioni

$$
\frac{\partial x_{1}}{\partial y_{1}}=\frac{\partial x_{2}}{\partial y_{2}} \quad \frac{\partial x_{1}}{\partial y_{2}}=-\frac{\partial x_{2}}{\partial y_{1}}
$$

Al sistema formato dalle (12) e (1:3) se ne può eridentemente sostituire uno equivalente in cui la i risulti indipendente da $x_{1}\left(\mathrm{o} d \mathrm{da} x_{2}\right)$ : onde al posto della (13) potremo supporre di arere un'equazione del tipo

$$
\psi\left(x_{2}, y_{1} y_{2}\right)=\varkappa
$$

Le equazioni (14) si possono allora scrivere

$$
\left.\begin{array}{c}
\frac{\partial \rho}{\partial y_{1}} \frac{\partial \psi}{\partial x_{2}}-\frac{\partial p}{\partial x_{2}} \frac{\partial \psi}{\partial y_{1}}-\frac{\partial \varphi}{\partial x_{1}} \frac{\partial \psi}{\partial y_{2}}=0 \\
-\frac{\partial \psi}{\partial y_{2}} \frac{\partial \psi}{\partial x_{2}}-\frac{\partial p}{\partial x_{1}} \frac{\partial \psi}{\partial y_{1}}+\frac{\partial \varphi}{\partial x_{2}} \frac{\partial \psi}{\partial y_{2}}=0
\end{array}\right\}
$$

e queste dovranno essere soddisfatte per $x_{1}, x_{2}, y_{1}, y_{2}$ soddisfacenti a (12).

Le (16) sono un sistema di equazioni lincari alle derivate partali per la $\psi\left(x_{2} y_{1} y_{2}\right)$; affinchè esso ammetta una soluzione non costante occorre che sia completo $\left(^{*}\right)$. Formiamo l'alternata delle due equazioni, ricordando ehe nell'eseguire questo calcolo occore pensare le rariabili $x_{1}, x_{2}, y_{1}, y_{2}$

(*) Pui essere ulile la consillerazione the sogue, la quale permelte di prevedero, diremo cosi, sinteticamente che it risultato del calcolo del testo che esprime che il sistema (l6) i completo, deve riportarci allequazione $0-0$. Si ammetta perciós che, come i dimostralo nel !. seguente, una jpersuperficie composta di superficie caratteristiche possa sempre essere frontiera comune per due funzioni $f(x, y)$ meromorfe l'una nel campo $p>0$, l'altra nel campo

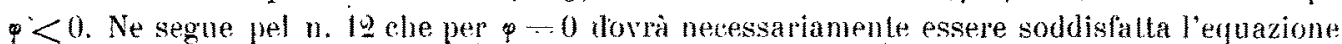

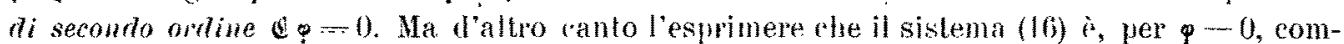

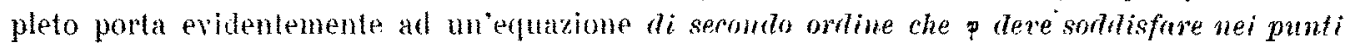
$\$=0$. Questr equazione non può quindi differire dallequatime $q=0$. 
legate dalla (12): otterremo l'equazione

$$
\begin{aligned}
& {\left[\Delta_{2}^{\prime \prime} p \cdot \frac{\partial p}{\partial x_{1}}-\left(\frac{\partial^{2}}{\partial x_{1} \partial y_{1}}+\frac{\partial^{2}}{\partial x_{2} \partial y_{2}}\right) \frac{\partial q}{\partial y_{1}}-\left(\begin{array}{c}
\partial^{2} \\
\partial x_{1} \partial y_{2}
\end{array}-\frac{\partial^{2} q}{\partial x_{2} \partial y_{1}}\right) \frac{\partial p}{\partial y_{2}}\right] \frac{\partial y}{\partial x_{3}}+}
\end{aligned}
$$

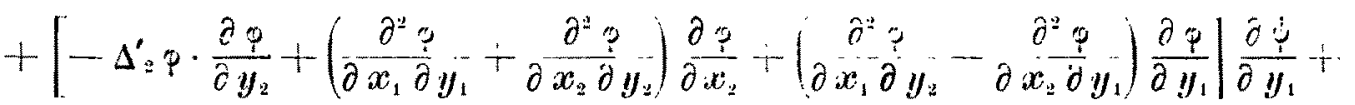

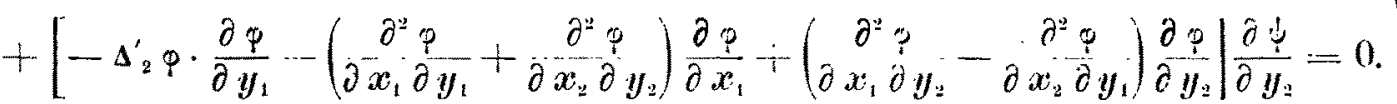

Ed affinchè questa equazione sia conseguenza di (16) occorrerì che il determinante dei coefficienti di (16) e (17) sia mullo: sviluppanto i calcoli si ottiene che deve essiere perciò - nei punti di (12) -

$$
\mathfrak{Q}:=0 \text {. }
$$

Inversamente, soldisfatta questa equazione, il sistema (16) è completo; e presa una qualungue sua soluzione quale primo membro di (15), Je (15) segano sulla varietà (19) un sistema semplicemente inlinilo di superficie caratteristiche. La simmetria rapporto alle variabili $x_{1} x_{2}$, e $y_{1} y_{2}$ del risultato indica che questo è indipendente dall'avere supposto la (12) risolubile rapporto a $x_{1}$. Il lemma è quindi pienamente dimostato.

14. Per provare ora che preso un pezzo suflicientemente piccolo di una ipersuperficie $S$ soddisfacente a (11) è sempre possibile costruire una funzione analitica esistente nellintorno di $S$ da una parte prefissata e che ha $S$ per frontiera noi dimostreremo che, almeno quando si suppone il campo che si considera sufticientemente piecolo, si può sempre immergere la semplice infinità di superficie caralteristiche costituenti $S$ in una doppia infinità lale che per ogni punto del campo passi una od una sola superlicie $S$ caratteristica. Costruiremo poi una funzione analitica $(--0)$ che si annulli sopra infinite delle superficie caratteristiche cosi costruite aventi per insieme limite tutte le superficie di $S$ : una tale funzione avrà evidentemente su $S$ tutti punti singolari essenziali.

Il primo punto è quasi evidente. Supponjamo che l'origine sia un punto di $S^{\prime}$ e che ad essa corrisponda il valore $\alpha=0$ del parametro $\%$ nelle (15). Supponiamo come precedentemente che il sistema (12), (15) sia nell'intorno dell'origine risolubile rapporto $x_{1}$ ed $x_{2}$ : avremo

$$
x_{1}=\Phi_{1}\left(y_{1} y_{2} \alpha\right) \quad x_{2}=\Phi_{2}\left(y_{1} y_{2} \alpha\right)
$$


$\Phi_{1}$ e $\Phi_{2}$ essendo funzioni armoniche coniugate di $y_{1} y_{2}$, e funzioni derivabili di $\alpha$; almeno in un certo campo attorno ai valori $y_{1}=y_{2}=\alpha=0$. Potremo anche scrivere:

$$
x=\Phi(y x)
$$

Ф essendo funzione analitica della variabile complessa $y$ e derivabile della variabile reale $\alpha$. Avremo inoltre

$$
\left|\frac{\partial x}{\partial x}\right|^{2}=\left(\frac{\partial \Phi_{1}}{\partial x}\right)^{2}+\left(\frac{\partial \Phi_{2}}{\partial \alpha}\right)^{2}=-\frac{\Delta_{1} \varphi}{\left[\frac{\partial(\varphi \psi)}{\partial\left(x_{1} x_{2}\right)}\right]^{2}}=0
$$

Consideriamo allora l'insieme di superficie caratteristiche

$$
x=\Phi(y \propto)+k \beta
$$

dove $\beta$ è una variabile reale, $k$ è un numero complesso $=0$, e, tale che il rapporto $\frac{\left(\frac{\partial x}{\partial \alpha}\right)_{y, \alpha=0}}{k}$ è ancora un numero complesso (non reale). Le (19) comprendono come caso particolare per $\beta=0$ le superficie (18): di più in ogni punto dell'intorno dell'origine passa una ed una sola superficie caratteristica (19). Basta osservare che le (19) equivalgono alle

$$
x_{2}-\Phi_{1}\left(y_{1} y_{2} \alpha\right)-k_{1} \beta=0 \quad x_{2}-\Phi_{2}\left(y_{1} y_{2} \alpha\right)-k_{2} \beta=0
$$

e che nel punto $x=y=\alpha=\beta=0$ si ha che il determinante funzionale di queste equazioni rapporto ad \% e \& è

$$
\left|\begin{array}{cc}
\partial \Phi_{1} & \partial \Phi_{2} \\
\partial \alpha & \partial \alpha \\
k_{1} & k_{2}
\end{array}\right|=\frac{\partial \Phi_{1}}{\partial \alpha} k_{2}-\frac{\partial \Phi_{2}}{\partial \alpha} k_{1}=k \mathfrak{J}\left[\frac{\left(\frac{\partial x}{\partial \alpha}\right)_{y, \alpha=3}}{k}\right]
$$

dore $\$(u)$ indica il coefficiente dell'immaginario di $\mu$ : e quindi per le ipotesi fatte su $k$ è certo $=0$.

Noi potremo quindi nell'intorno dell'origine determinare un campo $C$ interno al campo $x<0, y<p$ tale che per ogni punto appartenente a $O$ passa una ed una sola superficie caratteristica (19) corrispondente ad $\alpha<\sigma, \mid \beta<\sigma$; ed inversamente al ogni valore $\alpha<\sigma, \beta<\sigma$ corrisponde una superficie (19) i cui punti arenti coordinata $y$ minore in modulo a $a$ sono interni a $C$. $\grave{E}$ chiaro per continuità che le superficie $(19)$ per cui $\beta>0$ 
apparterranno ad una medesima delle regioni in cui $S$ divide lo spazio, ad es.: alla $\varphi>0$, le superficie per cui $\beta<0$ apparterranno all'altra.

Passiamo ora alla costruzione della funzione $f(x y)$. Consideriamo perciò un insieme di punti $\left(\alpha_{n}, \beta_{n}\right)$ tale che sia sempre $\beta_{n}>0$ (o sempre $\left.\beta_{n}<0\right)$ e che l'insieme derivato di esso sia l'insieme $|\alpha| \leq \sigma, \beta=0$. Basta, perciò per es.: numerare al modo solito i valori razionali di $\alpha$ minori di $\sigma$ e prendere per $\alpha_{.,}$l'n-esimo punto in tale numerazione, per $\beta_{n}$ il valore $\frac{1}{n}$.

Consideriamo allora il prodotto infinito $\left({ }^{*}\right)$ :

$$
\Pi_{n}\left(\frac{\left.x-\Phi\left(y, \alpha_{n}\right)-k \beta_{n}\right)}{x-\Phi\left(y, \alpha_{n}\right)}\right) e^{p_{n}\left(x_{y}\right)}=\Pi_{n}\left(1-\frac{k}{n\left(x-\Phi\left(y, \alpha_{n}\right)\right)}\right) e^{p_{n}(x y)}
$$

dove

$$
\left.\begin{array}{c}
p_{n}(x y)=\frac{k}{n\left(x-\Phi\left(y, \alpha_{n}\right)\right)}+\frac{1}{2} \frac{k^{2}}{n^{2}\left(x-\Phi\left(y, \alpha_{n}\right)\right)^{2}}+\cdots \\
\cdots+\frac{1}{n} \frac{k^{n}}{n^{n}\left(x-\Phi\left(y, \alpha_{n}\right)\right)^{n}} \cdot
\end{array}\right\}
$$

$\grave{E}$ facile vedere che il prodotto (20) è uniformemente convergente in ogni campo $\Gamma$ appartenente a $C$ il quale non contenga alcun punto di $S$. Se ammettiamo per un momento tale convergenza, esso rappresenteri evidentemente una funzione analitica di $x$ ed $y$, nulla su tutte le superficie caratteristiche (19) corrispondenti ai valori $\alpha_{n}$ e $\beta_{n}$ di $\alpha$ e $\beta$; e quindi, come dicemmo, una funzione che esiste nel campo $\beta>0$ ossia $\varphi>0$, ed ha necessariamente $S$ come frontiera. E chiaro che si potrebbe coslruire una funzione analoga nel campo $?<0$ col porre $\beta_{n}=-\frac{1}{n}$; e con ciò resta dimostrato il nostro teorema.

Occorre quindi solo dimostrare la convergenza uniforme in un qualunque campo $\Gamma$ del prodotto (20). Intichiamo perciò con $d$ la minima distanza dei punti di $r$ da $S$. Sarà per un qualunque punto $\left(x_{0} y_{0}\right)$ di $r,\left|x_{0}-\Phi\left(y_{0} \alpha_{n}\right)\right| \geqslant d$ : infatti $\mid x_{0}-\Phi\left(y_{0}, x_{n}\right)$ rappresenta la distanza del punto $\left(x_{0} y_{0}\right)$ dal punto in cui il piano $y=y_{0}$ incontra la superficie $x=\Phi\left(y, \alpha_{n}\right)$, la quale appartiene tutta ad $S$. Fissato allora un numero $\approx$ piccolo a piacere, dividiamo i numeri $n$

(*) Il ragionamento che segue non ò che la ripetizione sotto forma luggermente diversa del classico ragionamento del Weienstrass relativo alla scomposizione di una funzione intera in fattori primarii. 
in due classi a seconda che $\left|\frac{k}{n d}\right| \geqslant 1-$ a oppure che $\left|\frac{k}{n d}\right|<1-\varepsilon$ : la prima conterrà solo un numero finito di valori di $n$ : poichè sarà $n \leq \frac{k}{(1-\varepsilon) d}$ : noi nel considerare il prodotto (20) lasceremo da parte questi valori di $n$. Per gli altri valori di. $n$ si ha

$$
\left|\frac{k}{n\left(x-\Phi\left(y, \alpha_{n}\right)\right)}\right| \leq\left|\frac{k}{n d}\right|<1-\varepsilon,
$$

quindi i fattori corrispondenti di $n$ in (20) non si annulleranno mai per $(x y)$ in $\Gamma$ : e per dimostrare che (20) converge uniformemente ad un limite $-=0$ basterà dimostrare la convergenza della serie dei logaritmi dei singoli termini. Ora per (21) tale serie è

$$
-\sum_{n}\left(\sum_{n=1}^{m=\infty} \frac{1}{n+m} \frac{k^{n+\cdots}}{n^{n+m}\left(x-1\left(y, \alpha_{n}\right)\right)^{n+m}}\right) .
$$

Ma per $(x y)$ in $r$ e

$$
\begin{aligned}
& \left|\sum_{1}^{\infty} \frac{1}{n+m} \frac{k^{n+m}}{n^{n+m}\left(x-\Phi\left(y, x_{, n}\right)\right)^{m+m}}\right|< \\
& <\left|\frac{1}{n^{m+1}} \frac{k^{\prime \prime+1}}{d^{\prime \prime+1}}\right| \sum_{0}^{\infty}\left|\frac{k^{m}}{n^{m} d^{m}}\right| \leq \\
& \leq \frac{1}{\varepsilon} n^{n+1} d^{n+1}
\end{aligned}
$$

onde segue che la serie dei moduli di (22) converge più rapidamente della. serie

$$
-\frac{1}{\varepsilon} \mathbf{y}(1-\varepsilon)^{n+1}=\frac{1}{\varepsilon^{i}}
$$

e quindi converge uniformemente in $r$.

Onde resta pienamente dimostrato il nostro enunciato.

15. Ove si interpretino i risultati degli studii precedenti come risultati relativi al sistema di equazioni alle derivate parziali cui soddisfa la parte reale di una funzione di due variabili complesse, si ottengono indicazioni non prive di valore. $\grave{\mathrm{E}}$ noto che, assegnare $\mathrm{i}$ valori di una tale funzione sopra l'ipersuperficie contorno di campo, è assegnare condizioni sovrabbon- 
danti; quali siano le condizioni cui devono soddisfare tali valori è un problema ancora assai oscuro non ostante gli sforzi di parecchi studiosi. Orbene, da quanto precede risulta che dette condizioni portano non solo sulla natura della funzione assegnata, ma anche e principalmente su quella dell'ipersuperficie considerata; in quanto che, se, per es., si suppone questa ipersuperficie analitica, non potrebhe assegnarsi su essa una fumzione non analitica a meno che l'ipersuperficie stessa non sodrlisfaccia, rispetto al campo che essa limita, alle condizioni del n. 12

Ottobre 1909. 